\title{
Hadronic Physics in Peripheral Heavy Ion Collisions
}

\author{
A. A. Natale \\ Instituto de Física Teórica, Universidade Estadual Paulista, \\ Rua Pamplona 145, 01405-900, São Paulo, SP, Brazil
}

Received on 9 March, 2002

\begin{abstract}
We discuss the production of hadronic resonances in very peripheral heavy ion collisions, where the ions collide with impact parameter larger than twice the nuclear radius and remain intact after the collision. We compare the resonance production through two-photon and double Pomeron exchange, showing that when we impose the condition for a peripheral interaction the $\gamma \gamma$ process dominates over the Pomeron interaction, due to the short range propagation of this last one. We also discuss the observation of light resonances through the subprocess $\gamma \gamma \rightarrow R \rightarrow \gamma \gamma$, which is a clean signal for glueball candidates as well as one way to check the existence of a possible scalar $\sigma$ meson.
\end{abstract}

\section{Introduction}

Collisions at relativistic heavy ion colliders like the Relativistic Heavy Ion Collider RHIC/Brookhaven and the Large Hadron Collider LHC/CERN (operating in its heavy ion mode) are mainly devoted to the search of the Quark Gluon Plasma. In addition to this important feature of heavy-ion colliders, we will also have ultraperipheral collisions with impact parameter $b>2 R_{A}$, where $R_{A}$ is the nuclear radius, and where the ions remain intact after the collision.

These interactions will be mostly of electromagnetic origin: two-photon $(\gamma \gamma)$ or photonuclear processes $(\gamma A)$. Due to the very strong photon field of each charge $Z$ accelerated ion, the photon luminosity will be quite high. In the case of RHIC final states produced in the two-photon process with an invariant mass up to a few $\mathrm{GeV}$ will appear at large rates. Above this scale the photon luminosity drops very fast. At LHC a final state with a mass almost two orders of magnitude larger can still be produced at reasonable rates. The variety of processes that can be studied in heavy ion peripheral collisions have been extensively reviewed recently [1].

The fact that hadronic resonances $(R)$ could be produced at large rates in peripheral heavy ion collisions was already discussed many years ago [2]. Perhaps this may be one of the most interesting studies to be performed at RHIC, because the machine will serve as a factory of light hadrons in $\gamma \gamma$ and $\gamma A$ reactions. Vector resonances will appear at huge rates in photonuclear reactions [3], as well as scalar and pseudoscalar resonances in two-photon processes [4]. We will particularly focus our attention on the production of scalar and pseudoscalar resonances through the $\gamma \gamma$ process.

Two-photon physics at $e^{+} e^{-}$colliders provided for a long time a lot of information on hadronic resonances [5]. The two-photon process is very important because it involves the electromagnetic coupling of the resonance, and its knowledge with high precision is very useful, for instance, to unravel the possible amount of mixing in some glueball candidates [6], complementing the information obtained through the observation of hadronic decays. Another interesting study is the possible production of a light scalar meson $(\sigma)$ whose existence has been discussed for several years [7]. We stress again that the advantage of relativistic heavy ion colliders is that the photon luminosity for two-photon physics is orders of magnitude larger than the one at available $e^{+} e^{-}$machines.

We will discuss the production of light hadronic resonances in ultra-peripheral heavy ion collisions. We will show that the process $\gamma \gamma \rightarrow R \rightarrow \gamma \gamma$, see Fig. (1), can be observed for many resonances above or at the same level of the background. The main background is the continuum reaction $\gamma \gamma \rightarrow \gamma \gamma$, this one will be discussed as well as some other background contributions. Double Pomeron exchange may also compete with the $\gamma \gamma$ physics, we will point out that this contribution is not important for very heavy ions.

The distribution of this review is the following: In Section II we present the distribution functions for photons and Pomerons in the ion, and discuss some of the approximations to obtain realistic cross sections. We compare $\gamma \gamma$ processes with the ones initiated by double Pomeron exchange. In Section III we discuss the $\gamma \gamma \rightarrow R \rightarrow \gamma \gamma$ reaction and its background in the case 
of some glueball candidates and in the case of a possible scalar $\sigma$ meson. Section IV contains our conclusions.

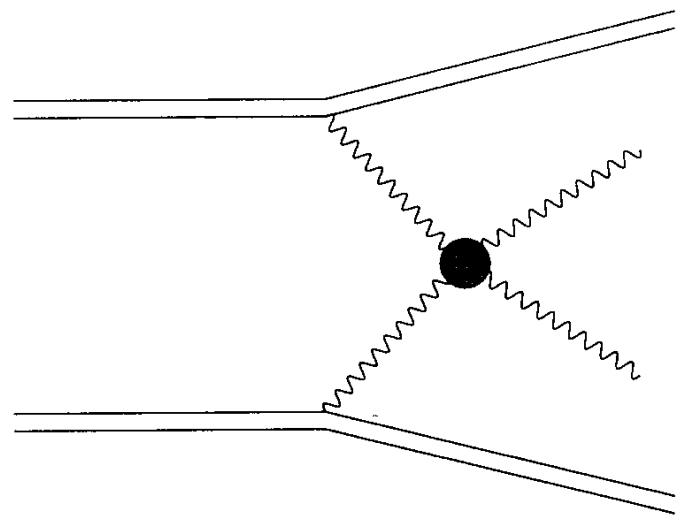

Figure 1. Diagram for $\gamma \gamma$ fusion in a peripheral heavy-ion collision. The blob represents a continuum or resonant process leading to a two-photon final state.

\section{Two-photon and double Pomeron exchange processes}

\section{A. Distribution functions}

The photon distribution in the nucleus can be described using the equivalent-photon or WeizsäckerWilliams approximation in the impact parameter space. Denoting by $F(x) d x$ the number of photons carrying a fraction between $x$ and $x+d x$ of the total momentum of a nucleus of charge $Z e$, we can define the two-photon luminosity through

$$
\frac{d L}{d \tau}=\int_{\tau}^{1} \frac{d x}{x} F(x) F(\tau / x)
$$

where $\tau=\hat{s} / s, \hat{s}$ is the square of the center of mass (c.m.s.) system energy of the two photons and $s$ of the ion-ion system. The total cross section of the process $A A \rightarrow A A \gamma \gamma$ is

$$
\sigma(s)=\int d \tau \frac{d L}{d \tau} \hat{\sigma}(\hat{s})
$$

where $\hat{\sigma}(\hat{s})$ is the cross-section of the subprocess $\gamma \gamma \rightarrow$ $X$.

There remains only to determine $F(x)$. In the literature there are several approaches for doing so, and we choose the conservative and more realistic photon distribution of Ref. [8]. Cahn and Jackson [8], using a prescription proposed by Baur [9], obtained a photon distribution which is not factorizable. However, they were able to give a fit for the differential luminosity which is quite useful in practical calculations:

$$
\frac{d L}{d \tau}=\left(\frac{Z^{2} \alpha}{\pi}\right)^{2} \frac{16}{3 \tau} \xi(z),
$$

where $z=2 M R \sqrt{\tau}, M$ is the nucleus mass, $R$ its radius and $\xi(z)$ is given by

$$
\xi(z)=\sum_{i=1}^{3} A_{i} e^{-b_{i} z}
$$

which is a fit resulting from the numerical integration of the photon distribution, accurate to $2 \%$ or better for $0.05<z<5.0$, and where $A_{1}=1.909, A_{2}=12.35$, $A_{3}=46.28, b_{1}=2.566, b_{2}=4.948$, and $b_{3}=15.21$. For $z<0.05$ we use the expression (see Ref. [8])

$$
\frac{d L}{d \tau}=\left(\frac{Z^{2} \alpha}{\pi}\right)^{2} \frac{16}{3 \tau}\left(\ln \left(\frac{1.234}{z}\right)\right)^{3} .
$$

The condition for realistic peripheral collisions $\left(b_{\min }>\right.$ $\left.R_{1}+R_{2}\right)$ is present in the photon distributions showed above.

The processes that we shall discuss can also be intermediated by the diffractive subprocess $P P \rightarrow X$, where $P$ is the Pomeron.

In the case where the intermediary particles exchanged in the nucleus-nucleus collisions are Pomerons instead of photons, we can follow closely the work of Müller and Schramm [10] and make a generalization of the equivalent photon approximation method to this new situation. So the cross section for particle production via two Pomerons exchange can be written as

$$
\sigma_{A A}^{P P}=\int d x_{1} d x_{2} f_{P}\left(x_{1}\right) f_{P}\left(x_{2}\right) \sigma_{P P}\left(s_{P P}\right),
$$

where $f_{P}(x)$ is the distribution function that describe the probability for finding a Pomeron in the nucleus with energy fraction $x$ and $\sigma_{P P}\left(s_{P P}\right)$ is the subprocess cross section with energy squared $s_{P P}$. In the case of inclusive particle production we use the form given by Donnachie and Landshoff [11]

$$
f_{P}(x)=\frac{1}{4 \pi^{2} x} \int_{-\infty}^{-(x M)^{2}} d t\left|\beta_{A P}(t)\right|^{2}\left|D_{P}\left(t ; s^{\prime}\right)\right|^{2},
$$

where $D_{P}\left(t ; s^{\prime}\right)$ is the Pomeron propagator [12]

$$
D_{P}(t ; s)=\frac{\left(s / m^{2}\right)^{\alpha_{P}(t)-1}}{\sin \left(\frac{1}{2} \pi \alpha_{P}(t)\right)} \exp \left(-\frac{1}{2} i \pi \alpha_{P}(t)\right),
$$

with $s$ the total squared c.m. energy. The Regge trajectory obeyed by the Pomeron is $\alpha_{P}(t)=1+\varepsilon+\alpha_{P}^{\prime} t$, where $\varepsilon=0.085, \alpha_{P}^{\prime}=0.25 \mathrm{GeV}^{-2}$ and $t$ is a small exchanged four-momentum square, $t=k^{2}<<1$, so the Pomeron behaves like a spin-one boson. The term in the denominator of the Pomeron propagator, $\left[\sin \left(\frac{1}{2} \pi \alpha_{P}(t)\right)\right]^{-1}$, is the signature factor that express the different properties of the Pomeron under $\mathrm{C}$ and $\mathrm{P}$ conjugation. At very high c.m. energy this factor falls very rapidly with $\mathbf{k}^{2}$, whose exponential slope is given 
by $\alpha_{P}^{\prime} \ln \left(s / m^{2}\right), m$ is the proton mass, and it is possible to neglect this $\mathbf{k}^{2}$ dependence,

$$
\sin \frac{1}{2} \pi\left(1+\varepsilon-\alpha_{P}^{\prime} \mathbf{k}^{2}\right) \approx \cos \left(\frac{1}{2} \pi \varepsilon\right) \approx 1 .
$$

If we define the Pomeron range parameter $r_{0}$ as

$$
r_{0}^{2}=\alpha_{P}^{\prime} \ln \left(s / m^{2}\right),
$$

the Pomeron propagator can be written as

$$
\left|D_{P}\left(t=-\mathbf{k}^{2} ; s\right)\right|=\left(s / m^{2}\right)^{\varepsilon} e^{-r_{0}^{2} \mathbf{k}^{2}} .
$$

Since we are interested in the spatial distribution of the virtual quanta in the nuclear rest frame we are using $t=-\mathbf{k}^{2}$.

The nucleus-Pomeron coupling has the form [11]

$$
\beta_{A P}(t)=3 A \beta_{0} F_{A}(-t),
$$

where $\beta_{0}=1.8 \mathrm{GeV}^{-1}$ is the Pomeron-quark coupling, $A$ is the atomic number of the colliding nucleus, and $F_{A}(-t)$ is the nuclear form factor for which is usually assumed a Gaussian expression (see, e.g., Drees et al. in [13])

$$
F_{A}(-t)=e^{t / 2 Q_{0}^{2}}
$$

where $Q_{0}=60 \mathrm{MeV}$.

Performing the $t$ integration of the distribution function in Eq.(7) we obtain

$$
\begin{aligned}
f_{P}(x) & =\frac{\left(3 A \beta_{0}\right)^{2}}{(2 \pi)^{2} x}\left(\frac{s^{\prime}}{m^{2}}\right)^{2 \varepsilon} \int_{-\infty}^{-(x M)^{2}} d t e^{t / Q_{0}^{2}} \\
& =\frac{\left(3 A \beta_{0} Q_{0}\right)^{2}}{(2 \pi)^{2} x}\left(\frac{s^{\prime}}{m^{2}}\right)^{2 \varepsilon} \exp \left[-\left(\frac{x M}{Q_{0}}\right)^{2}\right] .
\end{aligned}
$$

The total cross section for a inclusive particle production is obtained with the above distribution and also with the expression for the subprocess $P P \rightarrow X$ as prescribed in Eq.(6).

\section{B. Is double Pomeron exchange a background for $\gamma \gamma$ processes?}

The double Pomeron exchange producing a final state $X$ have matrix elements with the same angular structure as the $\gamma \gamma$ one. This is easy to observe within the Donnachie and Landshoff model [12]. For instance, in this model to compute the cross section of the subprocess $P P \rightarrow R$ it is assumed that the Pomeron couples to the quarks of the resonance $(R)$ like a isoscalar photon [12]. This means that the subprocess cross section of $P P \rightarrow R$ can be obtained from suitable modifications on the cross section for $\gamma \gamma \rightarrow R$, and it will differ only by the coupling constant and a form factor describing the phenomenological Pomeron-quark coupling. Therefore it is natural to ask if the Pomeron process is a background to the two-photon process, and when it has to be added to the calculation of a specific process.

In general the double Pomeron exchange is not a background for the two-photon process and this is easy to understand. The Pomeron contrarily to the photon does not propagate at large distances, actually its propagator has a range parameter $r_{0}$ defined in Eq.(10). When we impose the condition for ultra peripheral collisions (i.e. the nuclei do not physically collide) the cross section diminishes considerably.

In Eq.(6) the cases where the two nuclei overlap are not excluded. To enforce the realistic condition of a peripheral collision it is necessary to perform the calculation taking into account the impact parameter dependence, $b$. It is straightforward to verify that in the collision of two identical nuclei the total cross section of Eq.(6) is modified to [10]

$$
\frac{d^{2} \sigma_{A A}^{P P \rightarrow X}}{d^{2} b}=\frac{Q^{\prime 2}}{2 \pi} e^{-Q^{\prime 2} b^{2} / 2} \sigma_{A A}^{P P},
$$

where $\left(Q^{\prime}\right)^{-2}=\left(Q_{0}\right)^{-2}+2 r_{0}^{2}$. The total cross section for inclusive processes is obtained after integration of Eq.(14) with the condition $b_{\min }>2 R_{A}$ in the case of identical ions.

Another way of to exclude events due to inelastic central collisions is through the introduction of an absortion factor computed in the Glauber approximation [14]. This factor modifies the cross section in the following form

$$
\begin{aligned}
\frac{d \sigma_{A A}^{g l}}{d^{2} b} & =\frac{d \sigma_{A A}^{P P \rightarrow R}}{d^{2} b} \exp \left[-A^{2} b \sigma_{0} \int \frac{d Q^{2}}{(2 \pi)^{2}} F_{A}^{2}\left(Q^{2}\right) e^{i Q b}\right] \\
& =\frac{d \sigma_{A A}^{P P \rightarrow R}}{d^{2} b} \exp \left[-A^{2} b \sigma_{0} \frac{Q_{0}^{2}}{4 \pi} e^{-Q_{0}^{2} b^{2} / 4}\right]
\end{aligned}
$$

where $\sigma_{0}$ is the nucleon-nucleon total cross section, whose value for the different energy domains that we shall consider is obtained directly from the fit of Ref. [15]

$$
\sigma_{0}=X s^{\epsilon}+Y_{1} s^{-\eta_{1}}+Y_{2} s^{-\eta_{2}},
$$

with $X=18.256, Y_{1}=60.19, Y_{2}=33.43, \epsilon=0.34$, $\eta_{1}=0.34, \eta_{2}=0.55, F_{A}\left(Q^{2}\right)=e^{-Q^{2} / 2 Q_{0}^{2}}$ and we exemplified Eq.(15) for the case of resonance production, i.e., $\sigma_{A A}^{P P \rightarrow R}$ is the total cross section for the resonance production to be discussed in the sequence. The integration in Eq.(15) is over all impact parameter space

We compared the rates for double Pomeron exchange and two-photon production of several final states like resonances, a pair of pions and a hadron cluster of invariant mass $M_{X}$. The details of the calculations can be found in Ref. [16] and here we will describe part of the results of that work.

In Table I we compare the cross sections for resonance production through the processes $\gamma \gamma, P P \rightarrow R$. 


\begin{tabular}{lccccc} 
Meson & $M_{R}$ & $\Gamma_{(R \rightarrow \gamma \gamma)}$ & $R H I C_{\gamma \gamma}$ & $L H C_{\gamma \gamma}$ & $R H I C_{\mathcal{P P}}$ \\
\hline$\pi^{0}$ & 135 & $8 \times 10^{-3}$ & 7.1 & 40 & 0.05 \\
$\eta$ & 547 & 0.463 & 1.5 & 17 & 0.038 \\
$\eta^{\prime}$ & 958 & 4.3 & 1.1 & 22 & 0.04 \\
$\eta_{c}$ & 2979 & 6.6 & $0.32 \times 10^{-2}$ & 0.5 & $0.47 \times 10^{-4}$ \\
$\eta_{c}^{\prime}$ & 3605 & 2.7 & $0.36 \times 10^{-3}$ & 0.1 & $0.34 \times 10^{-5}$ \\
$\eta_{b}$ & 9366 & 0.4 & $0.13 \times 10^{-7}$ & $0.37 \times 10^{-3}$ & $0.11 \times 10^{-10}$
\end{tabular}

Table I. Cross sections for resonance production through photon-photon $(\gamma \gamma)$ and double-Pomeron $(P P)$ processes. For $R H I C, \sqrt{s}=200 \mathrm{GeV} /$ nucleon, we considered ${ }^{238} \mathrm{U}$ ion and for $L H C, \sqrt{s}=6300 \mathrm{GeV} /$ nucleon, the nucleus is ${ }^{206} \mathrm{~Pb}$. The cross sections are in mbarn. Rates computed with the geometrical cut $b>2 R_{A}$.

\begin{tabular}{lcc} 
Meson & $\sigma_{A A}^{g l} / \sigma_{A A}^{\mathcal{P P} \rightarrow R}(L H C)$ & $\sigma_{A A}^{g l} / \sigma_{A A}^{\mathcal{P P} \rightarrow R}(R H I C)$ \\
\hline$\pi^{0}$ & $3.54 \times 10^{-3}$ & $1.5 \times 10^{-2}$ \\
$\eta$ & $3.58 \times 10^{-3}$ & $1.47 \times 10^{-2}$ \\
$\eta^{\prime}$ & $3.46 \times 10^{-3}$ & $1.5 \times 10^{-2}$ \\
$\eta_{c}$ & $3.47 \times 10^{-3}$ & $1.32 \times 10^{-2}$ \\
$\eta_{c}^{\prime}$ & $3.61 \times 10^{-3}$ & $1.5 \times 10^{-2}$ \\
$\eta_{b}$ & $3.5 \times 10^{-3}$ & $1.45 \times 10^{-2}$
\end{tabular}

Table II. Ratios of cross sections for diffractive resonance production calculated with the Glauber absorption factor to the one with the geometrical cut in the collision of ${ }^{238} \mathrm{U}$ for energies available at RHIC $(\sqrt{s}=200 \mathrm{GeV} / \mathrm{nucleon})$, and collisions of ${ }^{206} \mathrm{~Pb}$ for energies available at $\mathrm{LHC}(\sqrt{s}=6.300 \mathrm{GeV} /$ nucleon$)$.

\begin{tabular}{lcccc} 
Nucleus & $\sqrt{s}$ & $\sigma_{A A}^{\mathcal{P P} \rightarrow R}$ & $\sigma_{A A}^{g l^{\mathcal{P P}}}$ & $\sigma_{\gamma \gamma}$ \\
\hline $\mathrm{Au}(A=197)$ & 100 & 0.044 & $0.55 \times 10^{-3}$ & 2.4 \\
$\mathrm{Ca}(A=40)$ & 3500 & 0.043 & $0.39 \times 10^{-3}$ & 0.14 \\
$\mathrm{Si}(A=28)$ & 200 & $0.34 \times 10^{-2}$ & $0.15 \times 10^{-3}$ & $0.69 \times 10^{-2}$ \\
$\mathrm{Si}(A=28)$ & 100 & $0.22 \times 10^{-2}$ & $0.12 \times 10^{-3}$ & $0.39 \times 10^{-2}$
\end{tabular}

Table III: Cross section for $\pi^{0}$ production for different ions and at different energies. The energies are in $\mathrm{GeV} / \mathrm{nucleon}$ and the cross sections in mbarn. $\sigma^{P P \rightarrow R}$ is the cross section computed with the geometrical cut and $\sigma^{g l}$ is the one with the absorption factor.

Table II shows the ratios of cross sections for diffractive resonance production calculated with the Glauber absorption factor to the one with the geometrical cut. The exclusion of central collisions through the Glauber absorption factor is stronger than the one with the geometrical cut. Table III shows the $\pi^{0}$ production for different ions and at different energies. We observe that the rates for double Pomeron exchange becomes closer to the two-photon rates when we go to lighter ions.

The production of a cluster of particles through double Pomeron exchange is also dominated by the $\gamma \gamma$ process (see Fig. (1) of Ref. [16]).

In general we may say that for very heavy ions the double Pomeron exchange gives cross sections one order of magnitude (or more) below the two-photon process. This changes when we collide lighter ions. As we go down from large charge ions to smaller ones the effect of Pomeron physics increases and it dominates the electromagnetic physics, as happens in the proton case.
The fact that the Pomeron has a short range parameter is not the only fact that counts when we analyze each specific process. It must be also remembered that the Pomeron couples to light and heavy quarks differently. Apart from kinematics the differences in the rates of resonance production by photons and Pomerons increases between those resonances formed by light and heavy quarks, as seen in Table I.

\section{The reaction $\gamma+\gamma \rightarrow \gamma+\gamma$}

\section{A. The continuum process}

We are particularly interested in the photon-photon scattering because it can be a very clean signal for hadronic resonances like glueballs and the $\sigma$ meson. On the other hand this scattering is important by itself, and could probably be directly measured by the first time at RHIC, as predicted in Ref. [2]. 
The subprocess $\gamma \gamma \rightarrow \gamma \gamma$ up to energies of a few $\mathrm{GeV}$ is dominated by the continuous fermion box diagram, and is a background for the resonant $\gamma \gamma \rightarrow R \rightarrow$ $\gamma \gamma$ process. It was first calculated exactly by Karplus and Neuman [17] and De Tollis [18]. There are sixteen helicity amplitudes for the process and, due to symmetry properties, the number of independent amplitudes will be only five, that may be chosen to be $M_{++++}$, $M_{++--}, M_{+-+-}, M_{+--+}$and $M_{+++-}$. Where the + or - denotes the circular polarization values +1 and -1 . The remaining helicity amplitudes may be obtained from parity and permutation symmetry. Of these five helicity amplitudes, three are related by crossing, hence it is sufficient to give just three, which are presented in detail in Ref. [19].

The differential cross section of photon pair production from photon fusion, i.e. the box diagram, is

$$
\frac{d \sigma}{d \cos \theta}=\frac{1}{2 \pi} \frac{\alpha^{4}}{s}\left(\sum_{f} q_{f}^{2}\right)^{4} \sum|M|^{2} .
$$

$\theta$ is the scattering angle, $\alpha$ is the fine-structure constant, $q_{f}$ is the charge of the fermion in the loop and the sum is over the leptons $e$ and $\mu$ and the quarks $u, d$ and $s$, which are the relevant particles in the mass scale that we shall discuss. Another possible contribution to this continuum process comes from pion loops, which, apart from possible double counting, were shown to be negligible compared to the above one [22]. The second sum is over the sixteen helicity amplitudes, $M_{\lambda_{1} \lambda_{2} \lambda_{3} \lambda_{4}}$, where $\lambda_{1}$ and $\lambda_{2}$ correspond to polarizations of incoming photons and $\lambda_{3}$ and $\lambda_{4}$ for the outgoing photons. The matrix elements summed over the final polarizations and averaged over the initial polarizations is given by

$$
\begin{aligned}
\sum|M|^{2} & =\frac{1}{2}\left\{\left|M_{++++}\right|^{2}+\left|M_{++--}\right|^{2}+\left|M_{+-+-}\right|^{2}\right. \\
& \left.+\left|M_{+--+}\right|^{2}+4\left|M_{+++-}\right|^{2}\right\}
\end{aligned}
$$

We consider the scattering of light by light, that is, the reaction $\gamma \gamma \rightarrow \gamma \gamma$, in $\mathrm{Au}-\mathrm{Au}$ collisions for energies available at RHIC, $\sqrt{s}=200 \mathrm{GeV} /$ nucleon. We checked our numerical code reproducing the many results of the literature for the box subprocess, including asymptotic expressions for the low and high energies compared to the fermion mass present in the loop, and the peak value of the cross section (see, for instance, Ref. [20]).

In Fig. (2) the dependence of the ion-ion cross section with the cosine of the scattering angle $\theta$, in the two photon center-of-mass system, is shown for an invariant photon pair mass equal to $500 \mathrm{MeV}$. It is possible to observe that the cross section is strongly peaked in the backward direction, but is relatively flat out to $\cos \theta \approx 0.4$, where it starts rising very fast. It is symmetric with respect to $\theta$ and the same behavior is present in the forward direction.
It is possible to observe that the cross sections is strongly restricted when we introduce an angular cut. We will impose in all the calculations throughout this work a cut in the scattering angle equal to $|\cos \theta|=0.5$. This cut is conservative, but it will make possible to compare the cross section of the box diagram with rival processes, that will be discussed in the following sections, as well as it is enough to eliminate the effect of double bremsstrahlung (which dominates the region of $|\cos \theta| \approx 1)$. Finally, this kind of cut is totally consistent with the requirements proposed in Ref. [3]

The fermions that contribute in the box diagram are the leptons $e$ and $\mu$ and the quarks $u, d$ and $s$, which are important for the mass range that we are interested (heavier quarks will give insignificant contributions and the same is true for the charged weak bosons). We assumed for their masses the following values: $m_{e}=0.5109 \mathrm{MeV}, m_{\mu}=105.6584 \mathrm{MeV}, m_{u}=5$ $\mathrm{MeV}, m_{d}=9 \mathrm{MeV}$ and $m_{s}=170 \mathrm{MeV}$.

The electron gives the major contribution to the total result. The second most important contribution is due to the muon, but it is at least one order of magnitude smaller than the electron one. The $d$ and $s$ quark contribution (up to $\mathrm{O}(2 \mathrm{GeV})$ ) are smaller due to their masses and charges, because the process is proportional to $\left(q_{f}^{2}\right)^{4}$ where $q_{f}$ is their charge. Their contribution is also insignificant compared to the electron result.

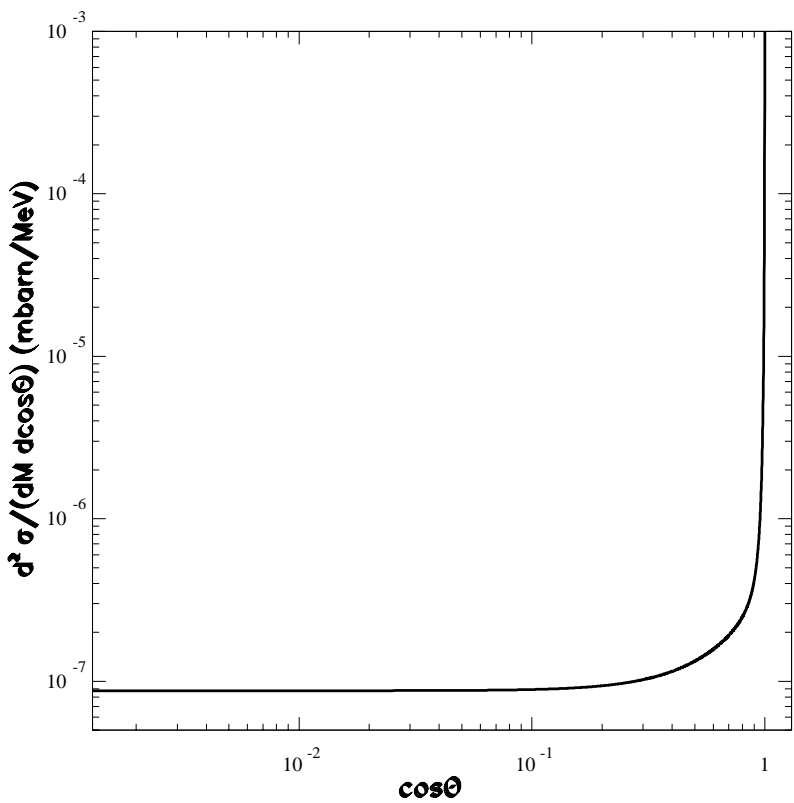

Figure 2. Angular distribution of $Z Z \rightarrow Z Z \gamma \gamma$ scattering at an invariant mass of $500 \mathrm{MeV}$. The scattering angle $\theta$ is in the photon pair center-of-mass system.

As discussed in Ref. [2] the $\gamma \gamma$ scattering can indeed be measured in peripheral heavy ion collisions. The cut in the angular distribution gives back to back photons in the central rapidity region free of the background. However, as we shall see in the sequence, there are gaps 
where the $\gamma \gamma \rightarrow \gamma \gamma$ process is overwhelmed by the presence of resonances like the $\eta, \eta^{\prime}$ and others. Even the broad $\sigma$ resonance could be of the order of the continuum process. Just to give one idea of the number of events, with a luminosity of $2.0 \times 10^{26} \mathrm{~cm}^{-2} \mathrm{~s}^{-1}[3]$ and choosing a bin of energy of $200 \mathrm{MeV}$, centered at the energy of $700 \mathrm{MeV}$ (which is free of any strong resonance decaying into two-photons), we have 1532 events/year assuming $100 \%$ efficiency in the tagging of the ions and photon detection.

\section{B. The process $\gamma \gamma \rightarrow R \rightarrow \gamma \gamma$ : glueballs}

Photon pair production via the box diagram is a background to $\gamma \gamma \rightarrow R \rightarrow \gamma \gamma$ process (or vice versa), both have the same initial and final states, and for this reason they can interfere one in another. Normally the interference between a resonance and a continuum process is unimportant, because on resonance the two are out of phase.

The total cross section for the elementary subprocess $\gamma \gamma \rightarrow R \rightarrow \gamma \gamma$ assuming a Breit-Wigner profile is

$$
\frac{d \sigma_{Z Z}^{\gamma \gamma}}{d M}=16 \pi \frac{d L}{d M} \frac{\Gamma^{2}(R \rightarrow \gamma \gamma)}{\left(M^{2}-m_{R}^{2}\right)^{2}+m_{R}^{2} \Gamma_{\text {total }}^{2}}
$$

where $M$ is the energy of the photons created by the collision of the ions. $\Gamma(R \rightarrow \gamma \gamma)\left(\equiv \Gamma_{\gamma \gamma}\right)$ and $\Gamma_{\text {total }}$ are the partial and total decay width of the resonance with mass $m_{R}$ in its rest frame.

We are going to discuss only $J=0$ resonances made of quarks as well of gluons. The reaction $\gamma \gamma \rightarrow \pi^{0} \rightarrow \gamma \gamma$ was already discussed many years ago [21], where it was claimed that the interference vanishes. This result was criticized by De Tollis and Violini [22], affirming (correctly) that the interference exists. However, as we will discuss afterwards, off resonance the interference is negligible. If the interference is neglected, Eq.(18) can be used and we show in Fig. (3) the result for some resonance production $\left(\eta, \eta^{\prime}, \eta(1440), f_{0}(1710)\right)$, whose invariant mass of the produced photon pair is between $500 \mathrm{MeV}$ and $2000 \mathrm{MeV}$. For comparison we also show the curve of the continuum process. It is possible to see in that figure the well pronounced peaks of the resonances $\eta$ and $\eta^{\prime}$. We assumed for their masses the values of $547.3 \mathrm{MeV}$ and $957.78 \mathrm{MeV}$, respectively, the $\eta$ total decay width is equal to $1.18 \mathrm{keV}$ and the $\eta^{\prime}$ one is equal to $0.203 \mathrm{MeV}$. Their partial decay width into photons are $0.46 \mathrm{keV}(\eta)$ and $4.06 \mathrm{keV}\left(\eta^{\prime}\right)$.

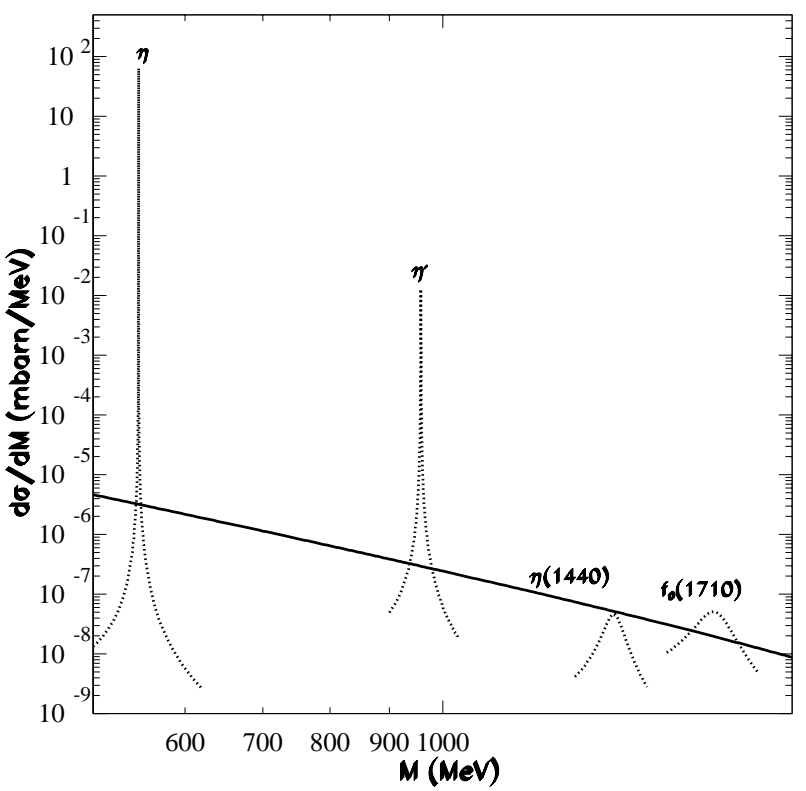

Figure 3. Invariant mass distribution of photon production (with the cut $|\cos \theta|<0.5$ ). The solid curve is for the box diagram, the dashed curves are due to the process $\gamma \gamma \rightarrow R \rightarrow \gamma \gamma$, where $R$ are the pseudoscalars resonances $\eta$ and $\eta^{\prime}$ and the glueballs candidates $\eta(1410)$ and $f_{0}(1710)$.

We restrict the analysis to the $J=0$ glueballs candidates $\eta(1440)$ and $f_{0}(1710)$. For the $\eta(1440)$ we used the mass and total decay width values of Ref. [23], $m_{R}=1405 \mathrm{MeV}, \Gamma_{\text {total }}=56 \mathrm{MeV}$, for the decay width into photons we use the value given in Ref. [24], $\Gamma_{\gamma \gamma}=5.4 \mathrm{keV}$. We see in Fig. (3) that the peak for this resonance is of the same order of the continuum process. For the other glueball candidate, $f_{0}(1710)$, the peak is clearly above the background. For this one we assumed the values listed in Ref. [23] of mass and total width, $m_{R}=1715 \mathrm{MeV} \Gamma_{\text {total }}=125 \mathrm{MeV}$, and for the two-photon decay width we adopted the value encountered by the ALEPH Collab. [25], $\Gamma_{\gamma \gamma}=21.25 \mathrm{keV}$. In all these cases the resonances can be easily studied in peripheral heavy ion collisions.

Off resonance we can expect a negligible contribution for the process $\gamma \gamma \rightarrow R \rightarrow \gamma \gamma$ and consequently the same for its interference with the continuum process. However, it is instructive to present a more detailed argument about why the interference can be neglected. In order to do so we are obliged to introduce a model to calculate the amplitudes for the process $\gamma \gamma \rightarrow R \rightarrow \gamma \gamma$. These amplitudes will be computed with the help of an effective Lagrangian for the pseudoscalar interaction with photons (the scalar case will be discussed in the next section), which is given by $g_{p} \varepsilon_{\lambda \mu \nu \rho} F^{\lambda \mu} F^{\nu \rho} \Phi_{p}$, where $g_{p}$ is the coupling of the photons to the pseudoscalar field $\Phi_{p}, \varepsilon_{\lambda \mu \nu \rho}$ is the antisymmetric tensor and $F^{\lambda \mu}$ is the electromagnetic field four-tensor.

The amplitudes for $\gamma \gamma \rightarrow \gamma \gamma$ intermediated by a 
pseudoscalar hadronic resonance are [22]:

$$
\begin{aligned}
& M_{++++}=\frac{2 \pi}{\alpha^{2}} F\left(\lambda r_{t}\right) \\
& M_{+-+-}=\frac{2 \pi}{\alpha^{2}} F\left(\lambda t_{t}\right), \\
& M_{+--+}=\frac{2 \pi}{\alpha^{2}} F\left(\lambda s_{t}\right), \\
& M_{+++-}=0, \\
& M_{++--}=-\frac{2 \pi}{\alpha^{2}}\left\{F\left(\lambda s_{t}\right)+F\left(\lambda t_{t}\right)+F\left(\lambda r_{t}\right)\right\}
\end{aligned}
$$

where $\alpha$ is the fine-structure constant, $\lambda=\left(m_{f} / m_{R}\right)^{2}$, and

$$
F(x)=16 x^{2} \frac{\Gamma_{\gamma \gamma}}{m_{R}}\left(4 x-1+i \frac{\Gamma_{\text {total }}}{m_{R}}\right)^{-1} .
$$

The presence of the fine-structure constant in Eq.(19) is a consequence of the fact that the amplitudes $M$ in these equations will be used in Eq.(17), so it is necessary to get the correct dependence of the partial cross section with this constant.

A numerical evaluation of the cross section using Eq.(19) (for the same resonances present in Fig. (3)) shows a totally negligible effect off resonance in comparison with the box contribution. On resonance the two processes are out of phase and the interference is absent. We can now proceed with an argument showing that the interference is not important. Let us assume that off resonance the processes are in phase, and for a moment we forget the $t$ and $u$ channels contribution in Eq.(19). The $s$ channel contribution can be written as $M_{2} /\left(s-m_{R}^{2}+i \Gamma_{R} m_{R}\right)$, and denoting the continuum contribution by $M_{1}$ we can write the following interference term

$$
\begin{aligned}
& 2 \frac{s}{\left(s-m_{R}^{2}\right)^{2}+\Gamma_{R}^{2} m_{R}^{2}}\left[\left(\operatorname{Re} M_{1} \operatorname{Re} M_{2}+\operatorname{Im} M_{1} \operatorname{Im} M_{2}\right)\right. \\
& \left.\left(s-m_{R}^{2}\right)+\left(\operatorname{Re} M_{1} \operatorname{Im} M_{2}-\operatorname{Im} M_{1} \operatorname{Re} M_{2}\right) \Gamma_{R} m_{R}\right] .
\end{aligned}
$$

We can verify that the term proportional to $s-m_{R}^{2}$ integrates to zero when integrated in a bin centered at $m_{R}^{2}$. With the second term the situation is different. If $\operatorname{Im} M_{1}$ or $\operatorname{Im} M_{2} \neq 0$ (assuming $\operatorname{Re} M_{1}$ and $\operatorname{Re} M_{2} \neq 0$ ) then there is a nontrivial interference. However, since we are dealing with $J=0$ amplitudes, the only nonvanishing helicity amplitudes are those in which the initial helicities and the final helicities are equal. Inspection of the $M_{++++}$and $M_{++--}$amplitudes of the box diagram (in the limit $m_{f} \ll m_{R}$ ) shows that they are purely real, and the same happens with $M_{2}$ (obtained from the $s$ channel contribution of Eq.(19)), resulting in a vanishing interference!

Of course, this analysis is model dependent. In particular, at the quark level the coupling $g_{p}$ has to be substituted by a triangle diagram, which may have a real as well as an imaginary part (see, for instance, Ref. [26]). However, this coupling is real for heavy quarks and its imaginary part is quite suppressed if the resonance couples mostly to light quarks $\left(m_{f} \ll m_{R}\right.$, what happens for the resonances that we are considering in the case of the $\mathrm{u}$ and $\mathrm{d}$ quarks, for the s quark the suppression is not so strong in the $\eta$ case, but we still have an extra suppression due to its electric charge). Finally, the interference does appear when we consider the amplitudes with the resonance exchanged in the $t$ and $u$ channels, but it is easy to see that they are kinematically suppressed and also proportional to the small value of the total decay width. If the total width is large (as we shall discuss in the next subsection) the interference cannot be neglected. Although the above argument has to rely on models for the low energy hadronic physics, we believe that the direct comparison between the resonant and the continuum processes, as presented in Fig. (3), is fairly representative of the actual result.

In Table IV we show the number of events above background for the $\eta, \eta^{\prime}$ and $f_{0}(1710)$ which, as shown in Fig. (3) are clearly above the box contribution. In the case of the $f_{0}(1710)$, as well as in the $\sigma$ meson case to be discussed in the next subsection, the decay of the resonance into a pair of neutral pions is present. A pair of neutral pions can also be produced in a continuous two photon fusion process. The rates for all the reactions discussed in this work can be modified if both neutral pions, no matter if they come from a resonance or a continuous process, are misidentified with photons. This accidental background can be easily isolated measuring its invariant mass distribution, and making a cut that discriminates a single photon coming from the processes that we are studying, from one that produced two neutral mesons (mostly pions) subsequently decaying into two photons. For example, in the case of the sigma meson (see next section) each one of the neutral pions from its much large hadronic decay should be misidentified. These pions would produce pairs of photons with a large opening angle $\phi$, where $\cos (\phi / 2)=\sqrt{1-4 m_{\pi}^{2} / m_{\sigma}^{2}}$. However, the calorimeters already in use in many experiments are able to distinguish between these two and single photon events with high efficiency (see, for instance, Ref. [27]).

\begin{tabular}{lc} 
particle & events /year \\
\hline$\eta$ & $7.44 \times 10^{5}$ \\
$\eta^{\prime}$ & $2.67 \times 10^{4}$ \\
$f_{0}(1710)$ & 42
\end{tabular}

Table IV. Number of events/year above background for the $\eta, \eta^{\prime}$ and $f_{0}(1710)$ resonances. 
There is also another accidental background which may cause problems to the measurement of the resonance decay into photons. This is the contribution from $\gamma$-Pomeron $\rightarrow \mathrm{V} \rightarrow \gamma \gamma X$. The vector mesons, $\mathrm{V}$, are produced with a $p_{T}$ distribution similar to the resonance production and at higher rates than some of the processes $\gamma \gamma \rightarrow R$, e.g. $\gamma-$ Pomeron $\rightarrow \omega$ rate is $10 \mathrm{~Hz}$ at RHIC [28], three orders of magnitude higher than for a similar mass meson of spin 0 or 2 . The $\omega$ branching ratio to three photons is $8.5 \%$. If a small $p_{T}$ photon from this decay is undetected, one is left with a low $p_{T}$ two-photon final state that could be taken for a lighter resonance. At higher masses, one also has $\phi \rightarrow \eta \gamma, \pi^{0} \gamma$, $K_{L} K_{S} \rightarrow \gamma X$ as well as $\gamma \gamma \rightarrow f_{2}(1270) \rightarrow \pi^{0} \pi^{0}$ and possibly copious production of $\rho(1450)$ and $\rho(1700)$ by $\gamma$-Pomeron interactions. Clearly a full simulation of all these background processes should be kept in mind when measuring two-photon final states.

\section{The process $\gamma \gamma \rightarrow R \rightarrow \gamma \gamma$ : the $\sigma$ meson}

The possible existence of light scalar mesons (with masses less than about $1 \mathrm{GeV}$ ) has been a controversial subject for roughly forty years. There are two aspects: the extraction of the scalar properties from experiment and their underlying quark substructure. Because the $J=0$ channels may contain strong competing contributions, such resonances may not necessarily dominate their amplitudes and could be hard to "observe". In such an instance their verification would be linked to the model used to describe them.

Part of the motivation to study the two-photon final states in peripheral heavy-ion collisions was exactly to verify if we can observe such scalar mesons in its $\gamma \gamma$ decay. Although this decay mode is quite rare, it has the advantage of not being contaminated by the strong interaction of the hadronic final states. In particular, it may allow to investigate the possible existence of the sigma meson. This meson is expected to have a mass between 400-1200 MeV and decay width between 300$500 \mathrm{MeV}$, decaying predominantly into two pions. Of course, another decay channel is into two photons, with the background discussed in the beginning of this Section.

Recently the E791 Collaboration at Fermilab found a strong experimental evidence for a light and broad scalar resonance, that is, the sigma, in the $D^{+} \rightarrow$ $\pi^{-} \pi^{+} \pi^{+}$decay [29]. The resonant amplitudes present in this decay were analyzed using the relativistic BreitWigner function given by

$$
B W=\frac{1}{m^{2}-m_{0}^{2}+i m_{0} \Gamma(m)},
$$

with

$$
\Gamma(m)=\Gamma_{0} \frac{m_{0}}{m}\left(\frac{p^{*}}{p_{0}^{*}}\right)^{2 J+1} \frac{J F^{2}\left(p^{*}\right)}{{ }^{J} F^{2}\left(p_{0}^{*}\right)},
$$

where $m$ is the invariant mass of the two photons forming a spin-J resonance. The functions ${ }^{J} F$ are the Blatt-Weisskopf damping factors [30]: ${ }^{0} F=1$ for spin 0 particles, ${ }^{1} F=1 / \sqrt{1+\left(r p^{*}\right)^{2}}$ for spin 1 and ${ }^{2} F=1 / \sqrt{9+3\left(r p^{*}\right)^{2}+\left(r p^{*}\right)^{4}}$ for spin 2. The parameter $r$ is the radius of the resonance $(\approx 3 \mathrm{fm})$ [31] and $p^{*}=p^{*}(M)$ the momentum of decay particles at mass $M$, measured in the resonance rest frame, $p_{0}^{*}=p_{0}^{*}\left(m_{R}\right)$. The Dalitz-plot of the decay can hardly be fitted without a $0^{++}(\sigma)$ resonance. The values of mass and total decay width found by the collaboration with this procedure are $478_{-23}^{+24} \pm 17 \mathrm{MeV}$ and $324_{-40}^{+42} \pm 21 \mathrm{MeV}$, respectively.

We will discuss if this resonance can be found in peripheral heavy-ion collisions through the subprocess $\gamma \gamma \rightarrow \sigma \rightarrow \gamma \gamma$. It is important to note that all the values related to the $\sigma$, like mass or partial widths, that can be found in the literature are very different and model dependent. In particular, we find the result of the E791 experiment very compelling and among all the possibilities we will restrict ourselves to their range of mass and total decay width, while we vary the partial width into two photons. For the $\sigma$ decay width into two photons we assume the values obtained by Pennington and Boglione, $3.8 \pm 1.5 \mathrm{keV}$ and $4.7 \pm 1.5 \mathrm{keV}$ [32], and the value of $10 \pm 6 \mathrm{keV}$ [33].

It has been verified in the case of $\pi \pi$ scattering that the use of a constant total width in the $\sigma$ resonance shape is not a good approximation [34]. In our case we will discuss the $\gamma \gamma \rightarrow \gamma \gamma$ process above the two pions threshold where the peculiarities of the broad resonance, basically due to the $\sigma$ decay into pions, are not so important. Of course, another reason to stay above $300 \mathrm{MeV}$ is that we are also far from the pion contribution to $\gamma \gamma$ scattering. In any case we also computed the cross section with a energy dependent total width $\Gamma(m) \simeq \Gamma_{0}\left(p^{*} / p_{0}^{*}\right)^{2 J+1}$, which, as shown by Jackson many years ago [35], is more appropriate for a quite broad resonance. The net effect is a slight distortion of the cross section shape with a small increase of the total cross section. Since this one is a negligible effect compared to the one that we will present in the sequence we do not shall consider it again.

Off resonance we can expect a negligible contribution for the process $\gamma \gamma \rightarrow R \rightarrow \gamma \gamma$ and consequently the same for its interference with the continuum process. This is true if the resonance has a small total decay width [19], but this is not the $\sigma$ case. To take into account the interference we must make use of a model 
to calculate the helicity amplitudes of the $\sigma$ meson exchange. Using the effective lagrangian $g_{s} F^{\mu \nu} F_{\mu \nu} \Phi_{s}$, where $g_{s}$ is the coupling of the photons to the scalar field $\Phi_{s}$ and $F^{\mu \nu}$ is the electromagnetic field tensor the following amplitudes comes out [19, 22]:

$$
\begin{aligned}
& M_{++++}=-\frac{2 \pi}{\alpha^{2}} F\left(\lambda r_{t}\right), \\
& M_{+-+-}=-\frac{2 \pi}{\alpha^{2}} F\left(\lambda t_{t}\right), \\
& M_{+--+}=-\frac{2 \pi}{\alpha^{2}} F\left(\lambda s_{t}\right), \\
& M_{+++-}=0, \\
& M_{++--}=-\frac{2 \pi}{\alpha^{2}}\left\{F\left(\lambda s_{t}\right)+F\left(\lambda t_{t}\right)+F\left(\lambda r_{t}\right)\right\}
\end{aligned}
$$

where $\alpha$ is the fine-structure constant, $\lambda=\left(m_{f} / m_{R}\right)^{2}$, and $r_{t}, s_{t}$ and $t_{t}$ are related with the standard Mandelstam variables $\mathrm{s}, \mathrm{t}$, and $\mathrm{u}$ by $r_{t}=\frac{1}{4} \frac{s}{m_{f}^{2}}, s_{t}=\frac{1}{4} \frac{t}{m_{f}^{2}}$ and $t_{t}=\frac{1}{4} \frac{u}{m_{f}^{2}}$

These amplitudes and the ones describing the fermion (with mass $m_{f}$ ) box diagram enter in the expression for the differential cross section of photon pair production from photon fusion $d \sigma / d \cos \theta=$ $(1 / 2 \pi)\left(\alpha^{4} / s\right)\left(\sum_{f} q_{f}^{2}\right)^{4} \sum|M|^{2}$, to give the total cross section of Fig. (4). We verified that the interference is destructive.

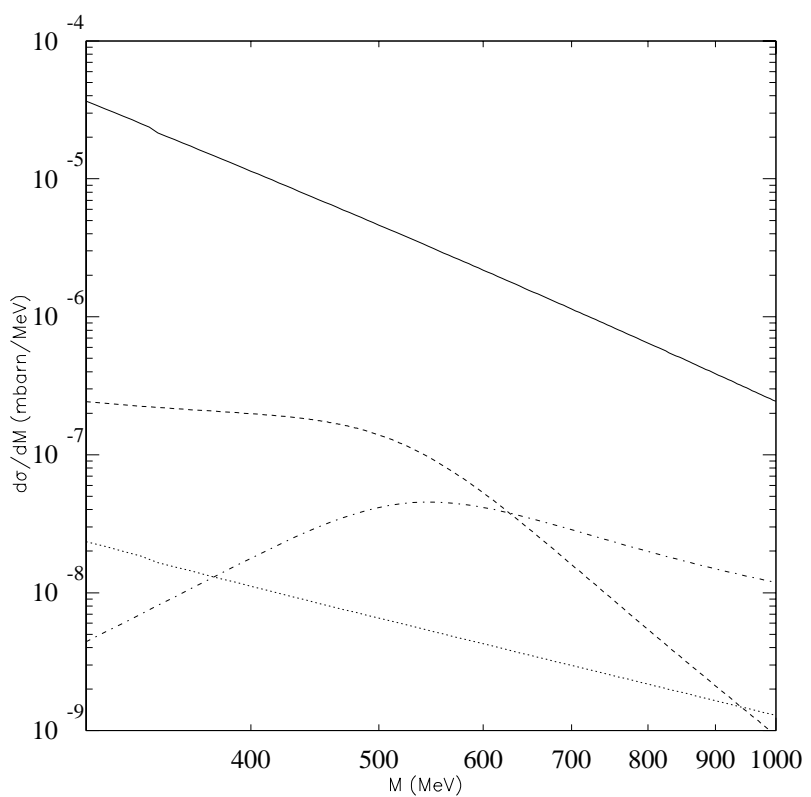

Figure 4. Invariant mass distribution of photon pair production. The solid curve is due to box diagram only, the dashed one is due to the process $\gamma \gamma \rightarrow \sigma \rightarrow \gamma \gamma$ in the Breit-Wigner approximation, the dash-dotted is the scalar contribution of Eq.(21), and the dotted one is the continuum Pomeron process $P P \rightarrow \gamma \gamma$. In all cases $\Gamma_{\gamma \gamma}=4.7 \mathrm{keV}$, and the angular cut is equal to $-0.5<\cos \theta<0.5$.

The effective Lagrangian model used to compute the $\sigma$ contribution to the photon pair production gives a larger cross section than the calculation with the BreitWigner approximation at energies above $M \approx 600$ $\mathrm{MeV}$. It is dominated by the $s$ channel contribution. We consider the Breit-Wigner result as the best signal representation for the resonant process because we are using the E791 data and this one was fitted by a Breit-Wigner profile. The effective Lagrangian gives a nonunitary amplitude that overestimates the sigma production above $600 \mathrm{MeV}$ and shows the model dependence in the $\sigma$ analysis that we commented before. The Breit-Wigner profile is not a bad approximation as long as we stay above the two pions threshold and in the following we assume that the signal is giving by it (the dashed curve of Fig. (4)) and the background is giving by the box diagram result (the solid curve of Fig. (4)). Note that, due to the destructive interference, the actual measurement will give a curve below the solid curve of Fig. (4).

From the experimental point of view we would say that the reaction $\gamma \gamma \rightarrow \gamma \gamma$ has to be observed and any deviation from the continuum process must be carefully modeled until a final understanding comes out, with the advantage that the final state is not strongly interacting. Note that in this modeling the $\eta$ meson will contribute to $\gamma \gamma \rightarrow \gamma \gamma$ in a small region of momentum [19], even so it has to be subtracted in order to extract the complete $\sigma$ signal.

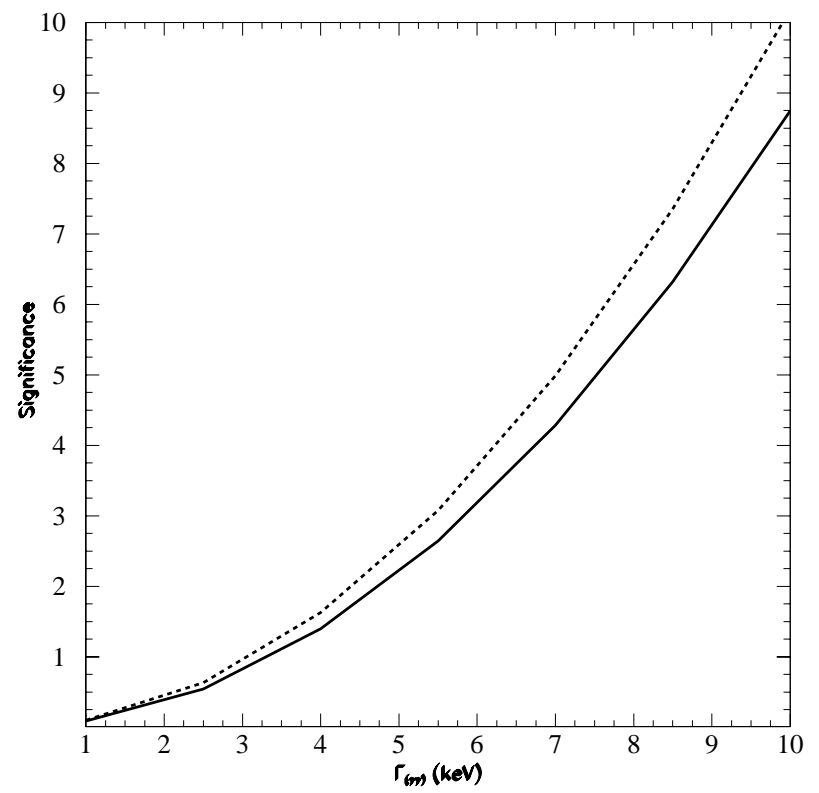

Figure 5. Significance as a function of decay width into two photons, $\Gamma_{\gamma \gamma}$, for a sigma meson with mass equal to 478 $\mathrm{MeV}$ and total decay width of $324 \mathrm{MeV}$. The solid curve was obtained integrating the cross sections in the interval $438<M<519 \mathrm{MeV}$, the dashed one in the interval $300<M<800 \mathrm{MeV}$. The signal and background are giving by the dashed (Breit-Wigner) and solid (box diagram) curves of Fig. (4) respectively. 
We changed the values of the $\sigma$ mass and total width around the central ones reported by the E791 Collaboration. We do not observed large variations in our result, but noticed that it is quite sensitive to variations of the partial decay width into photons. It is interesting to look at the values of the significance which is written as $\mathcal{L} \sigma_{\text {signal }} / \sqrt{\mathcal{L} \sigma_{\text {back }}}$ and characterizes the statistical deviation of the number of the observed events from the predicted background. The significance as a function of the two photons decay width of the sigma meson, with mass equal to $478 \mathrm{MeV}$ and total decay width of 324 $\mathrm{MeV}$, is shown in Fig. (5), were we used a luminosity of $\mathcal{L}=2.0 \times 10^{26} \mathrm{~cm}^{-2} \mathrm{~s}^{-1}$ at RHIC and assumed one year of operation. The significance is above $2 \sigma$ $95 \%$ confidence level limit for two photon decay width greater than $4.7 \mathrm{keV}$, while for a $5 \sigma$ discovery criteria can be obtained with $\Gamma_{\gamma \gamma}>7.5 \mathrm{keV}$. The numbers in Fig. (5) were computed with the signal given by the Breit-Wigner profile, and the background by the pure box diagram. The solid curve was obtained integrating the cross sections in the range of experimental mass uncertainty $438<M<519 \mathrm{MeV}$, while the dashed curve resulted from the integration in the interval $300<M<800 \mathrm{MeV}$. Note that there is no reason, a priori, to restrict the measurement to a small bin of energy. This choice will depend heavily on the experimental conditions. Therefore, for values of $\Gamma_{\gamma \gamma}$ already quoted in the literature the sigma meson has a chance to be seen in its two photon decay mode. The discovery limits discussed above refer only to a statistical evaluation. Our work shows the importance of the complete simulation of the signal and background including an analysis of possible systematic errors that may decrease the significance.

\section{Conclusions}

Peripheral collisions at relativistic heavy ion colliders provide an arena for interesting studies of hadronic physics. One of the possibilities is the observation of light hadronic resonances, which will appear quite similarly to the two-photon hadronic physics at $e^{+} e^{-}$machines with the advantage of a huge photon luminosity peaked at small energies [1]. Due to this large photon luminosity it will become possible to discover resonances that couple very weakly to the photons [4].

The double Pomeron exchange may be a background for the two-photon processes. We have shown that in general the Pomeron contribution does not compete with the $\gamma \gamma$ one, in the case of very heavy ions, after imposing the condition for a ultra-peripheral collision. However it must be stressed that this comparison has to be performed for each specific final state, due to the different Pomeron coupling to several particles. If the collision involves heavy ions and the final state we are looking at does not have a large coupling to the Pomeron we can affirm that double Pomeron exchange can be neglected in the evaluation of production rates. In the case of light ions Pomeron processes are competitive with the two-photons ones, and dominate the cross sections for very light ions.

We discussed the peripheral reaction $A A \rightarrow A A \gamma \gamma$. This process is important because it may allow for the first time the observation of the continuous subprocess $\gamma \gamma \rightarrow \gamma \gamma$ in a complete collider physics environment. This possibility only arises due to enormous amount of photons carried by the ions at the RHIC energies.

The continuous subprocess is described by a fermionic box diagram calculated many years ago. We computed the peripheral heavy ion production of a pair of photons, verifying which are the most important contributions to the loop, which turned out to be the electron at the energies that we are working, and established cuts that not only ensure that the process is peripheral as well as eliminate most of the background. After the cut is imposed we still have thousands of photons pairs assuming $100 \%$ efficiency in tagging the ions and detecting the photons.

The continuous $\gamma \gamma \rightarrow \gamma \gamma$ subprocess has an interesting interplay with the one resulting from the exchange of a resonance. We discuss the resonance production and decay into a photon pair. This is a nice interaction to observe because it involves only the electromagnetic couplings of the resonance. Therefore, we may say that it is a clean signal of resonances made of quarks (or gluons) and its measurement is important because it complements the information obtained through the observation of purely hadronic decays. It may also unravel the possible amount of mixing in some glueball candidates [6]. We discuss the interference between these process and compute the number of events for some specific cases.

The possibility of observing resonances that couple weakly to the photons is exemplified with the $\sigma$ meson case. This meson, whose existence has been for many years contradictory, gives a small signal in the reaction $\gamma \gamma \rightarrow \sigma \rightarrow \gamma \gamma$. However its effects may be seen after one year of data acquisition, providing some clue about this elusive resonance. Using values of mass, total and partial widths currently assumed in the literature, we compute the full cross section within a specific model and discuss the significance of the events. Our work shows the importance of the complete simulation of the signal and background of these processes including an analysis of possible systematic errors, indicating that 
two photon final states in peripheral collisions can be observed and may provide a large amount of information about the electromagnetic coupling of hadrons.

\section{Acknowledgments}

Most of this work has been done in collaboration with C. G. Roldão. I would like to thank I. Bediaga, C. Dib, R. Rosenfeld and A. Zimerman for many valuable discussions. This research was supported by the Conselho Nacional de Desenvolvimento Científico e Tecnológico $(\mathrm{CNPq})$, by Fundacão de Amparo à Pesquisa do Estado de São Paulo (FAPESP) and by Programa de Apoio a Núcleos de Excelência (PRONEX).

\section{References}

[1] G. Baur, K. Hencken, D. Trautmann, S. Sadovsky, and Y. Kharlov, hep-ph/0112211 Phys. Rep., in press; G. Baur, hep-ph/0112239; C. A. Bertulani and G. Baur, Phys. Rep.163, 299 (1988); G. Baur, J. Phys. G24, 1657 (1998); S. Klein and E. Scannapieco, hepph/9706358 (LBNL-40457); J.Nystrand and S. Klein, hep-ex/9811997 (LBNL-42524); C. A. Bertulani, nuclth/0011065, nucl-th/0104059; J. Rau, b. Müller, W. Greiner, and G. Soff, J. Phys. G: Nucl. Part. Phys. 16, 211 (1990); M. Greiner, M. Vidović, J. Rau, and G. Soff, J. Phys. G. 17, L45 (1990); M. Vidović, M. Greiner, C. Best, and G. Soff, Phys. Rev. C 47, 2308 (1993); M. Greiner, M. Vidović, and G. Soff, Phys. Rev. C47, 2288 (1993)

[2] G. Baur and C. A. Bertulani, Nucl. Phys. A505, 835 (1989).

[3] S. Klein and J. Nystrand, Phys. Rev. C60, 014903 (1999); J. Nystrand and S. Klein (LBNL-41111) nuclex/9811007, in Proc. Workshop on Photon Interactions and the Photon Structure eds. G. Jarlskog and T. Sjöstrand, Lund, Sweden, Sept., 1998; J. Nystrand, nucl-th/0112055; C. A. Bertulani and F. S. Navarra, nucl-th/0107035.

[4] A. A. Natale, Phys. Lett. B362, 177 (1995); Mod. Phys. Lett. A9, 2075 (1994).

[5] V. M. Budnev, I. F. Ginzburg, G. V. Meledin, and V. G. Serbo, Phys. Rept. 15, 181 (1974); V. P. Andreev, Nucl. Phys. Proc. Suppl. 96, 98 (2001).

[6] F. E. Close and A. Kirk, Eur. Phys. J. C21, 531 (2001).

[7] N. A. Tornqvist and M. Roos, Phys. Rev. Lett. 76, 1575 (1996); M. R. Pennington, Talk at the Workshop on Hadron Spectroscopy (WHS99), Frascati (March, 1999), hep-ph/9905241; N. A. Tornqvist, Summary talk of the conference on the sigma resonance (Sigmameson 2000), Kyoto (June, 2000), hep-ph/0008136.

[8] R. N. Cahn and J. D.Jackson, Phys. Rev. D42, 3690 (1990).

[9] G. Baur, in Proc. CBPF Intern. Workshop on Relativistic Aspects of Nuclear Physics, Rio de Janeiro, 1989, edited by T. Kodama et al. (World Scientific, Singapore, 1990)
[10] B. Müller and A. J. Schramm, Nucl. Phys. A523, 677 (1991).

[11] A. Donnachie and P. V. Landshoff, Phys. Lett. B191, 309 (1987); Nucl. Phys. B 303, 634 (1988).

[12] A. Donnachie and P. V. Landshoff, Nucl. Phys. B244, 322 (1984); BB267, 690 (1985).

[13] E. Papageorgiu, Phys. Rev. D40, 92 (1989); Nucl. Phys. A498, 593c (1989); M. Grabiak et al., J. Phys. G15, L25 (1989); M. Drees, J. Ellis, and D. Zeppenfeld, Phys. Lett. B223, 454 (1989); M. Greiner, M. Vidovic, J.Rau, and G. Soff, J. Phys. G17, L45 (1991); B. Müller and A. J. Schramm, Phys. Rev. D42, 3699 (1990); J. S. Wu, C. Bottcher, M. R.Strayer, and A. K. Kerman, Ann. Phys. 210, 402 (1991).

[14] V. Franco and R. J. Glauber, Phys. Rev. 142, 1195 (1966).

[15] C. Caso et al. (Particle Data Group), Eur. Phys. J. C3, 1 (1998).

[16] C. G. Roldão and A. A. Natale, Phys. Rev. C61, 064907 (2000).

[17] R. Karplus and M. Neuman, Phys. Rev. 83, 776 (1951).

[18] B. De Tollis, Nuovo Cimento 32, 757 (1964); 351182 (1965).

[19] A. A. Natale, C. G. Roldão, and J. P. V. Carneiro, Phys.Rev. C65, 014902 (2002).

[20] V. B. Berestetskii, E. M. Lifshitz, and L. P. Pitaevskii, in Quantum Electrodynamics, (Editions ButterworthHeinemann, Oxford, 1996), vol.4, pp. 571.

[21] V. N. Oraevskii, Sov. Phys. JETP 12, 730 (1961); M. Y. Han and S. Hatsukade, Nuovo Cimento 21, 119 (1961).

[22] B. De Tollis and G. Violini, Nuovo Cimento A41, 12 (1966).

[23] D. E. Groom et al. (Particle Date Group), Eur. Phys. J. C15, 1 (2000).

[24] F. E. Close, G. R. Farrar, and Z. Li, Phys. Rev. D55, 5749 (1997).

[25] ALEPH Collaboration, R. Barate et al., Phys. Lett. B472, 189 (2000)

[26] S. F. Novaes, Phys. Rev. D27, 2211 (1983).

[27] LAPP Collaboration, D. Alde et al., Z. Phys. C36, 603 (1987).

[28] S. Klein and J. Nystrand, Phys. Rev. C60, 014903 (1999).

[29] E791 Collaboration, E. M. Aitala et al., Phys. Rev. Lett. 86, 770 (2001).

[30] J. M. Blatt and V. F. Weisskopf, in Theoretical Nuclear Physics, John Wiley \& Sons, New York, 1952.

[31] ARGUS Collaboration, H. Albrecht et al., Phys. Lett. B308, 435 (1993).

[32] M. Boglione and M. R. Pennington, Eur. Phys. J. C9, 11 (1999). 
[33] A. Courau et al., Nucl. Phys. B271, 1 (1986).

[34] F. Sannino and J. Schechter, Phys. Rev. D52, 96 (1995); M. Harada, F. Sannino, and J. Schechter,
Phys. Rev. D54, 1991 (1996); Phys. Rev. Lett. 78, 1603 (1997)

[35] J. D. Jackson, Nuovo Cimento 34, 1644 (1964). 